\title{
An Empirical Research on Teaching Dynamic Classifiers in Chinese International Education
}

\author{
Yanan $\mathrm{Gao}^{1, \mathrm{a} *}$ and Xu Gao ${ }^{2, \mathrm{~b}}$ \\ ${ }^{1}$ Shenyang Aerospace University No.37Ave Daoyi, Shenyang, China \\ ${ }^{2}$ Liaoning Academy of Cultural and Artistic Institute No.68 Ave Shenyang, Shenyang, China \\ agaoyanan05111031@163.com, b937456696@qq.com \\ *The corresponding author
}

Keywords: Chinese classifiers; International education; Teaching methods; Empirical analysis

\begin{abstract}
Aiming at the important and difficult problem in the Chinese international education, the paper proposes effective strategies for teaching Chinese dynamic classifiers. They are general teaching method of bridge-experiential, teaching sequence of individual after measuring classifiers and verbal after noun classifiers, and the polysemy classifiers strategy of the semantic network. It not only expounds the theoretical basis of each method and strategy, but also gives specific class operation procedures, and makes field investigations of their application effects.
\end{abstract}

\section{Introduction}

Since Martion Martini first discussed Chinese classifiers in 1653, its research has been more than 300 years history. This kind of words has been paid much attention by international typology linguistics, foreign linguistics and national linguistics. Compared with the theoretical research results, the research results of classifiers in Chinese international education are not very rich. They mainly concentrated in two aspects, one is to explore the internal rationale of the use modern Chinese classifier, providing theoretical guidance and explanation for foreign students (Xiao Guozheng 2004, Fu Xue Feng 2005). The other one is to analyze and diagnose the errors produced by the foreign students in the acquisition of Chinese classifiers, and put forward the teaching suggestions (Zhao Xuejing 2009, Tang Ying 2010). The teaching of classifiers are the key and difficult problems in the international education of Chinese. However, there are few practical teaching methods and strategies according to characteristics of Chinese classifiers. Therefore, this paper takes dynamic classifiers as study objects, which have been derived from the verb and retained certain dynamic characteristics. It puts forward the effective teaching mode, teaching order and strategy, at same time points out specific teaching methods and operation steps to make the students learn Chinese classifiers better, enhances efficiency finally of teaching Chinese as the second language.

\section{Bridge - experiential Teaching Method}

Theoretical Basis. Scaffolding instruction is one of constructivist teaching methods. The so-called "scaffolding" teaching means that through the support of the holder, the task of management learning is gradually transferred from the teacher to the students themselves, then the scaffolding is withdrawn and students complete the construction of the knowledge. By activating the structure related to the teaching content, the scaffolding method builds the support for the effective connection and transition between each knowledge point, and reduces the cognitive difficulty of the new knowledge.

Dynamic classifiers originate from verbs, which are the results of further grammaticalization of verbs. At the same time, the source verbs of dynamic classifiers are basically action verbs with strong action. They can generally be recognized by some actions of the body. Therefore, we can try to build an action verbs as a teaching scaffolding in teaching classifiers, helping students to learn the dynamic classifier through the simulation of the action and the experience of the meaning 
construction process.

Based on the principles of experience philosophy and cognitive linguistics, Wang Yin (2005)proposes that the category, concept and mind of human beings were formed on the basis of physical experience. Therefore, in the international teaching of Chinese, the mental simulation process of the formation of dynamic classifiers can be displayed through actual actions, so that students can recognize them through the multichannel movements of kinesthetic (student action) and vision (student watching action) and hearing (student hearing).

Concrete Operation Steps of Bridge-experiential Teaching Method. We take the dynamic classifier "chuan" as an example to elaborate the bridge experiential teaching method. Because the classifier "chuan" comes from the verb "to put things together", Firstly, we make the students construct the teaching scaffolding of the verb "chuan" in the mind.

Teacher: (showing pictures of sugarcoated haws on a stick on PPT) What is he stringing?

Student 1: Sugarcoated haws.

Teacher: (showing a picture of lamb shashlik on PPT) What is he doing?

Student 2: He is making a string of mutton.

Teacher: (teacher prepares several keys and a rope) string up the key, please.

Student 3: The student makes this action.

Then through verb "chuan", we introduce dynamic classifier "chuan" to help students understand the semantic construction process of it.

Teacher: Many verbs in Chinese can be changed into classifiers gradually. (the teacher pointed to the keys of the students) what we should say?

Student: Yi chuan yaoshi .

Teacher: What do we call a string of sugarcoated haws?

Student: Yi chuan tanghulu.

Teacher: what about a string of mutton?

Student: Yi chuan yangrou .

Finally, some exercises are designed to deepen the understanding. For example:

Multiple choice questions: yi chuan ( ) A.hua B. jiaoyin C. shengyin D. xianglian E. baozu F. wenti

Fill in the blanks: liang chuan ( ), yi da chuan ( ), yi chuan chuan( )

Bridge-experiential Teaching Effect of Method. In order to investigate the effectiveness of this teaching method, two parallel classes of Chinese primary 2 (82 persons) at Shenyang Aerospace University were selected for comparative research. We use the form of questionnaires, which include choosing answers, filling in the blank, lining up and making sentences. As shown in 1, The average result of class 1 which adopt this strategy is higher than the 2 class that teach routinely, especially for choosing answers, the correct rate of the bridge-experience teaching is up to $95.6 \%$.

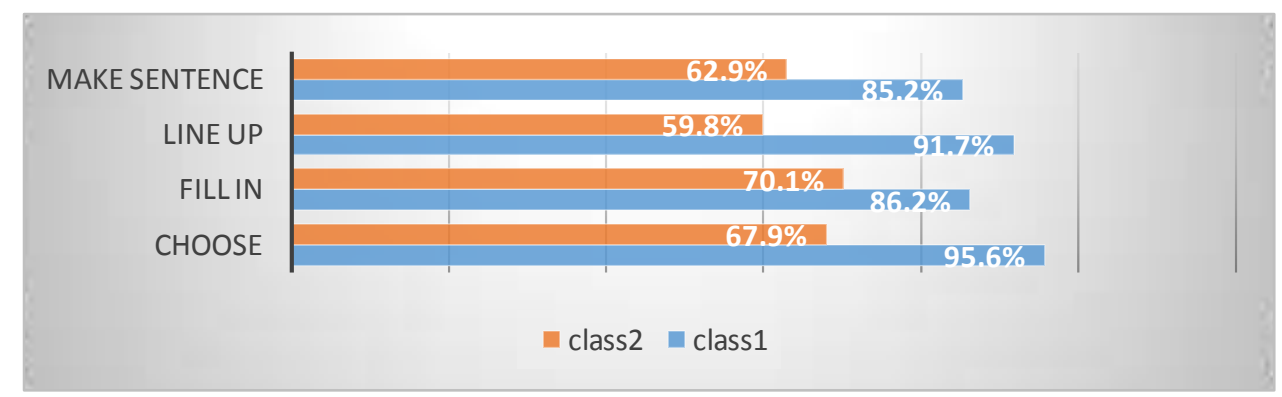

Figure 1. Finite

\section{Teaching Sequence of Individual after Measuring Classifiers, Verbal after Noun Classifiers.}

Theoretical Basis. S.P. Corder, (1967) put forward the inherent outline hypothesis. It is believed that the second language learners do not obey the teacher's teaching arrangement passively, but have their own intrinsic acquisition rules and order. American language teaching theorist S. Krashen also 
believes that the rules of language have a fixed natural order. He denotes the idea of comprehensible input that the entering language information is neither too difficult nor easy, and he uses $i+1$ to express it. He believes that the level of input language should be controlled in the degree of $i+1$, because i+2 learners are hard to understand, and i+0 can not develop their language ability. So teachers should consider the intrinsic acquisition order of the learners as much as possible.

In the framework of grammaticalization, Gao Shunquan (2012) discusses the acquisition order of teaching Chinese as a second language. It is considered that both grammaticalization and language acquisition are cognitive processes, they all follow the general law of human cognition. The order of grammaticalization from low to high is in great agreement with the order of acquisition. Through the study of the categorization of collective classifiers and the process of historical development, Zong Shouyun (2008) also draws a conclusion that process of classifiers' extending from near distance to long are basically consistent with the grammaticalization course of the classifiers.

Teaching Order of Dynamic Classifiers. Dynamic classifiers can be divided into two kinds: dynamic noun classifiers and dynamic verbal classifiers according to syntactic distribution. We believe that the dynamic noun classifiers should be taught before verbal ones. First of all, dynamic noun classifiers precede dynamic verbal classifiers. Both grammaticalization degree and frequency of usage are significantly higher than verbal words. Secondly, for the Chinese locals and Chinese learners, the noun classifiers are recognized ahead of the verbal classifiers. We asked five Chinese native speakers (Zhang Jiazi, Wei Xin, Yi Nayi, Liu Anna, Li Yan) and five foreign students studying in China (Lee Hsien / DPRK, Siminking / South Korea, Noda / Japan, Olia / Russia, Lin Wei / US) to say at least five classifiers quickly. They all answer noun classifiers such as "ge", "zhang" and "Bao" unexceptionally. Only in the guidance of the author, they can think of verbal ones.

The dynamic noun classifiers can be divided into four categories: individual classifiers, collective classifiers, and measure words. In the teaching of Chinese dynamic noun classifiers, the order of the measure words and collective classifiers should be preceded by the individual classifiers. Firstly, it is a comprehensible input and consistent with the Chinese development law. Because from the diachronic level, the measure words and collective classifiers are first emerged, and the individual classifiers are gradually developed on the basis of those two types of classifiers.

The Effect of this Teaching Order. A comparative study was conducted on two parallel classes (76 persons) of Chinese primary 1. We adopt to analysis that combine of qualitative and quantitative, such as test, questionnaire, interview and classroom observation. The data collected from two dimensions, including error rate and time-taken, In2, whether the error rate of the written or oral test class 1 used in this order is lower than the class 2 controlled normally. In 3, class1 lower significantly than class 2 , that is, teaching sequence of individual after measuring classifiers, verbal after noun classifiers not only improves the output accuracy to a certain extent to, but also increases the learning efficiency.

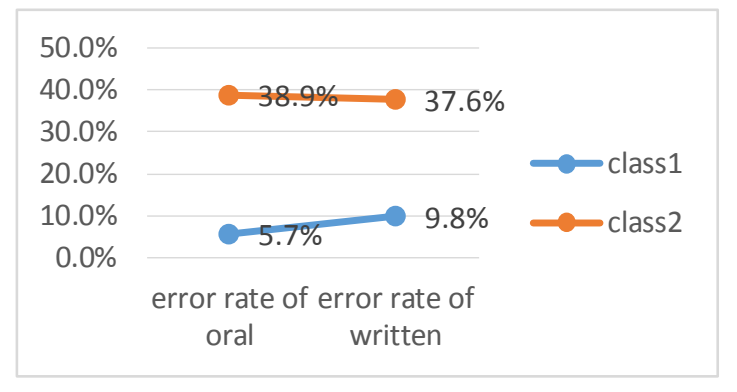

Figure 2.Finite

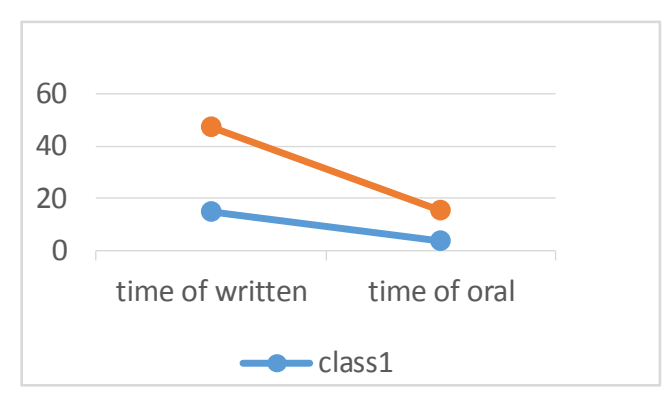

Figure 3. Finite

\section{The Semantic Network Strategy of Polysemous Dynamic Classifiers}

Theoretical basis. According to memory theory, memory is divided into two categories: mechanical memory and meaningful memory. The study shows that meaningful memory is superior 
to mechanical one in terms of comprehensiveness, accuracy, consolidation and speed. Moreover, the more coded of knowledge, the greater probability of entering long-term memory and the less chance of forgetting. Meanwhile, the information stored in the long-term memory is not isolated and chaotic, but ordered according to semantic classification. Ausubel, D.P. also points out the material that teachers provided to students should be meaningful and organized so that students can get a lot of system knowledge in a short time. In view of the above memory and learning theory, teachers should help students to establish the links between all kinds of meanings in teaching Chinese polysemous classifiers, and try to make full semantic coding for their uses. In the middle and advanced stages, it is necessary for teachers to make a summary of the complex usage of them, and classify and collate the discrete dynamic classifiers structure, so that students can form the semantic memory network in the mind.

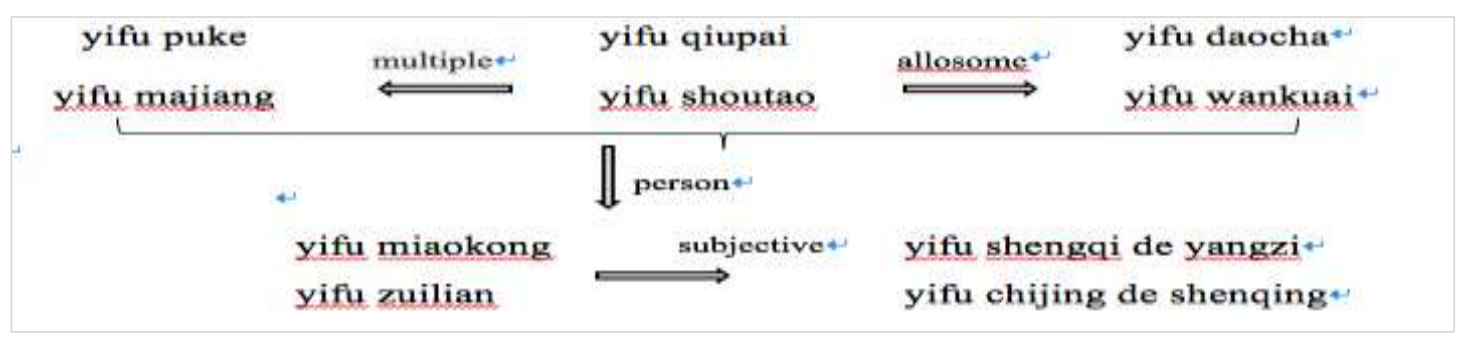

Figure 4. Finite

POLYSEMOUS dynamic Classifiers Teaching Example. The polysemous dynamic classifier "fu" can match both a pair of things, such as "a pair of earrings" and "a pair of racket", as well as a complete set of things, such as "a pair of chopsticks" and "a pair of mahjong". It can also combine with people's expressions, such as "yifu zuilian" and "yifu buxie de shenqing". Facing these various uses of the classifier "fu", Chinese teachers should strive to construct the semantic association between these usages so that the students can make meaningful memorization on the basis of understanding, and build the semantic network of the uses.

Study on the Effect of this Strategy. A comparative study of two intermediate Chinese parallel classes (a total of 84 people) from Shenyang Aerospace University was carried out to investigate the effectiveness of this teaching strategy. A continuous follow-up test was conducted by using the test questionnaire method which choose now, three months later and one year later. In 5, correct rate of class 1 adopted this strategy was maintained steadily, and higher than class 2 with the conventional teaching control, the correct rate remained above ninety percent after a year. That is, the semantic network strategy had a positive impact on the learning effect of polysemous dynamic classifiers and the retention of memory for a long time.

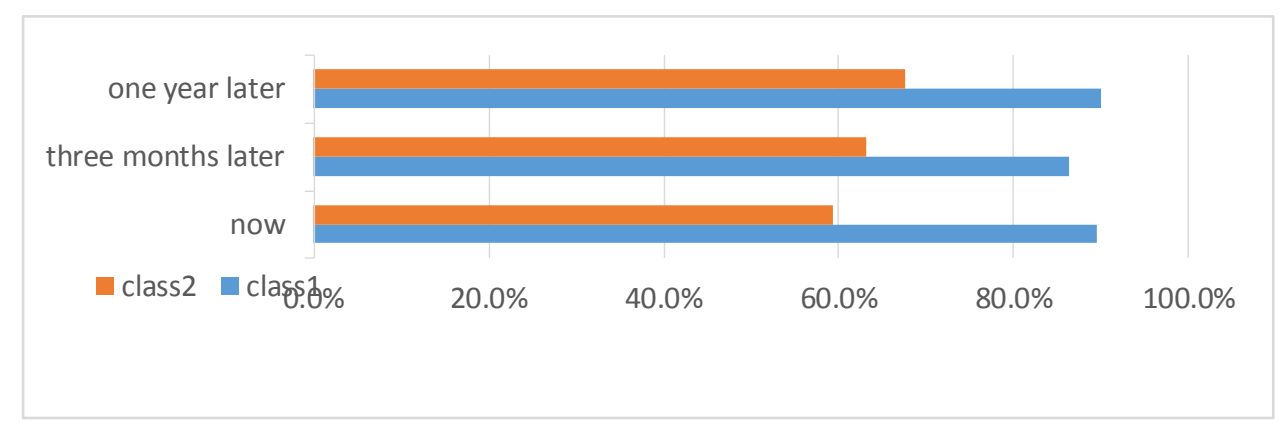

Figure 5. Finite

\section{Acknowledgements}

Liaoning provincial department of education project: Study on Chinese communication system of foreign students' in China from The Belt and Road countries(L201645). 


\section{Reference}

[1] A.Y. Aikhenvald, Classifiers: A Typology of Noun Categorization Device.Oxford: Oxford University Press, 2015.

[2] W. Bisang, Classifiers in East and Southeast Asian language Counting and beyond. L.S.Cheng, \& R. Sybesma, Bare and Not-So-Bare Nouns and the Structure of NP, Linguistic Inquiry, 30 (1999).

[3] Y. C. Chien, \& B. Lust, \& C. P. Chiang, Chinese Children's Comprehension of Count-Classifiers and Mass-Classifiers. Journal of East Asian Linguistics, 12(2013).

[4] H.H. Gao, A Study of Swedish speakers's Learning of Chinese Noun Classifiers, Nordic Association of Linguists, 33 (2010).

[5] M. Gerner, Verb Classifiers in East Asia, Functions of Language, 21(2015).

[6]C. R. Huang, \& A. Kathleen, Individuals, Kinds and Events: Classifier Coercion of Nouns. Language Sciences, 25(2013).

[7] J. Y. Kuo, \& M.D. Sera, Classifier Effects on Human Categorization: the Role of Shape Classifiers in Mandarin Chinese, Journal of East Asian Linguistics, 18(2009).

[8] G. Lakoff, \& M. Johnson, Philosophy in the flesh:the embody mind and its challenge to western thought. New York: Basic Books, 1999.

[9] W. D. Li, The Pragmatic Function of Numeral-Classifiers in Mandarin Chinese, Journal 10 of Pragmatics, 32(2016).

[10] X. P. Li, Numeral Classifiers in Chinese, Berlin:Mouton de Gruyter, 2013. 